08

\title{
Аналитическая модель атомно-слоевого осаждения пленок на 3D-структурах с высоким аспектным отношением
}

\author{
(C) А.В. Фадеев, А.В. Мяконьких, К.В. Руденко \\ Физико-технологический институт РАН, \\ 117218 Москва, Россия \\ e-mail: AlexVFadeev@gmail.com
}

(Поступило в Редакцию 13 июня 2017 г.)

\begin{abstract}
Предложена теоретическая модель, позволяющая предсказать пространственный профиль пленки, нанесенной на стенки высокоаспектной структуры (тренча) методом атомно-слоевого осаждения, в зависимости от параметров процесса осаждения. Модель позволяет рассчитать оптимальное время дозирования прекурсора, необходимое для конформного покрытия стенок тренча. Получена приближенная формула, описывающая осаждение пленок равной толщины на стенки тренча, включающая два асимптотических режима осаждения при различных соотношениях между коэффициентом прилипания молекул прекурсора и аспектным отношением структуры тренча.
\end{abstract}

DOI: 10.21883/JTF.2018.02.45416.2385

\section{Введение}

Метод атомно-слоевого осаждения (ALD) является наиболее перспективным методом однородного покрытия $3 D$-структур пленками нанометровой толщины [1]. Данный метод состоит в последовательном введении химически активных веществ (прекурсоров) в камеру реактора с промежуточной продувкой камеры. Молекулы каждого из вводимых прекурсоров химически не взаимодействуют между собой в газовой фазе, а взаимодействуют с поверхностью посредством необратимой химической реакции. Таким образом, реакции на поверхности прекращаются после того, как будут заняты все активные центры адсорбции, тем самым обеспечивая как однородность растущей пленки, так и жесткий контроль ее толщины. Несмотря на то, что метод ALD обладает самоограничением, для трехмерных структур важным параметром является транспорт прекурсора в зону проведения реакции. Параметры ALD процессов для большинства пленок оптимизированы лишь для случая плоской поверхности. В случае же выращивания пленок на $3 D$-структурах с высоким аспектным отношением (отношение глубины $H$ к ширине $W$ ) встает вопрос как о величине необходимой длительности экспозиции каждого из прекурсоров, так и от времени продувки реактора для получения однородного покрытия рассматриваемых структур. Большинство работ в этой области представляет собой численное моделирование методом Монте-Карло [см., например, 2,3]. Среди теоретических работ одной из наиболее известных моделей, описывающих рост пленки в круглых отверстиях, является работа [4]. Модель основана на ослаблении с глубиной кнудсеновского потока, текущего по трубе, относительно входного отверстия. Основными недостатками данной модели являются принятый равным единице коэффициент прилипания частиц к стенкам и пренебрежение отраженными потоками от дна круглого отвер- стия. Также в литературе представлены работы [5-7], основанные на кинетических уравнениях, описывающих как диффузионное течение газа в отверстии, так и реакции, описывающие убыль активных для реакции, с прекурсором узлов со временем. В этом подходе также не учитывается отраженный поток от дна круглого отверстия.

В настоящей работе предлагается теоретическая модель атомно-слоевого осаждения на поверхность тренча (канавки с прямоугольным профилем), основанная на кинетических уравнениях, определяющих степень заполнения элемента поверхности со временем подачи прекурсора. Потоки на стенки и дно тренча определяются согласно молекулярно-кинетической теории.

\section{1. Система кинетических уравнений, описывающих рост пленки}

Для определенности будем рассматривать рост пленки $\mathrm{HfO}_{2}$ методом ALD из тетраэтилметиламиногафния (ТЕМАН) и паров воды. Из-за большой молекулярной массы и низкого давления насыщенных паров по сравнению с парами воды плотность потока молекул ТЕМАН на несколько порядков меньше плотности потока воды. Оптимизация времени экспозиции ТЕМАН позволяет снизить расход дорогостоящего прекурсора и общее время процесса. Поэтому будем считать, что во время подачи второго прекурсора (воды) происходит полное насыщение реакции во всех областях рассматриваемой структуры. И далее будем рассматривать только взаимодействие первого прекурсора с поверхностью. Также предполагаем, что времени продувки достаточно для полного удаления из реактора как прекурсоров, так и продуктов их реакции с поверхностью. При указанных предположениях пространственная форма профиля (зависимость толщины осажденной пленки от координаты) 
растущей пленки будет однозначно определяться только первым прекурсором.

Рассмотрим характерные величины, соответствующие прекурсору ТЕМАН [1]. Температура ТЕМАН в газовой фазе составляет $T_{g}=120^{\circ} \mathrm{C}$, а температура поверхности $T_{s}=270^{\circ} \mathrm{C}$. Радиус молекулы ТЕМАН можно примерно оценить как $a_{\text {TEMAH }}=1 \mathrm{~nm}$. Для определения парциального давления прекурсора в газовой фазе примем, что в камеру реактора за $1 \mathrm{~s}$ подается $1 \mathrm{mg}$ ТЕМАН и $350 \mathrm{sccm}$ Ar. При этом суммарное давление в камере составляет $\sim 80$ mTorr. Тогда парциальное давление ТЕМАН можно оценить по формуле

$$
P_{\mathrm{TEMAH}}=\frac{P_{\Sigma} v_{\mathrm{TEMAH}}}{v_{\mathrm{TEMAH}}+v_{\mathrm{Ar}}}=0.08 \text { mTorr. }
$$

В результате длина свободного пробега для молекул ТЕМАН при указанных параметрах будет составлять

$$
\lambda=\frac{k T_{g}}{\sigma P}=\frac{k T_{g}}{\pi a_{\mathrm{TEMAH}^{2} P}} \Rightarrow \lambda_{\mathrm{TEMAH}}=0.016 m,
$$

что значительно больше характерной ширины тренча $W \sim 100 \mathrm{~nm}$, и следовательно, мы имеем дело с молекулярным режимом течения газа внутри тренча.

Обозначим за $n_{0}=\frac{d N_{0}}{d S}-$ поверхностную концентрацию прекурсора в адсорбированном монослое. Тогда число частиц, адсорбированных поверхностью, можно представить в виде

$$
d N=\Theta d N_{0}=\Theta n_{0} d S,
$$

где $\Theta=\frac{d N}{d N_{0}}-$ степень покрытия стенок тренча.

При получении кинетических уравнений, описывающих степень покрытия стенок тренча в зависимости от времени подачи прекурсора, были приняты следующие допущения:

- Распределение частиц над плоской поверхностью максвелловское.

- При ударе о незаполненную поверхность $(\Theta=0)$ частица может либо прилипнуть к поверхности с вероятностью $s$, либо отразиться с вероятностью $1-s$ по закону косинуса.

- При отражении от заполненной поверхности $(\Theta=1)$ вероятность отражения равна единице и происходит по закону косинуса.

- Учитывая, что вероятность осаждения второго монослоя в идеальном ALD процессе равна нулю, вероятность отражения частиц можно определить выражением

$$
p=\Theta+(1-\Theta)(1-s)=1-s(1-\Theta) .
$$

- При взаимодействии с поверхностью частица приобретает температуру поверхности.

- Пренебрегаем поверхностной диффузией частиц как во время подачи прекурсора, так и во время продувки реактора.
С учетом принятых предположений уравнение, описывающее изменение числа частиц $N$ адсорбированных элементом поверхности тренча площадью $d S$, можно записать в виде

$$
\frac{d N}{d t}=\left(\Phi_{e}+\Phi_{r}\right) p_{s}
$$

где $p_{s}=1-p$, а $\Phi_{e}$ и $\Phi_{r}$ - потоки частиц на площадку $d S$ тренча из газовой фазы и за счет отражений от других частей тренча соответственно, $p_{s}$ - вероятность прилипания частиц к указанному элементу поверхности. Учитывая, что $d \Phi=J d S$, и вводя соответствующие $\Phi_{e}$ и $\Phi_{r}$ плотности потоков частиц $J_{e}, J_{r}$, получим уравнение, описывающее изменение степени заполнения стенок тренча со временем подачи прекурсора:

$$
n_{0} \frac{d \Theta}{d t}=\left(J_{e}+J_{r}\right) s(1-\Theta) .
$$

\section{1. Плотность потока частиц на боковую поверхность тренча из газовой фазы $\left(J_{e s}\right)$}

Число частиц, падающих на элемент поверхности тренча, определяется как число ударов о поверхность площадью $d S$ за время $d t$ :

$$
d \hat{N}=d N_{v} \frac{v_{\perp} d t d S}{V}
$$

где $v_{\perp}-$ перпендикулярная площадке $d S$ компонента скорости частиц, $d N_{v}$ - число частиц, имеющих скорость в диапазоне от $\mathbf{v}$ до $\mathbf{v}+d \mathbf{v}$. Умножая и деля на

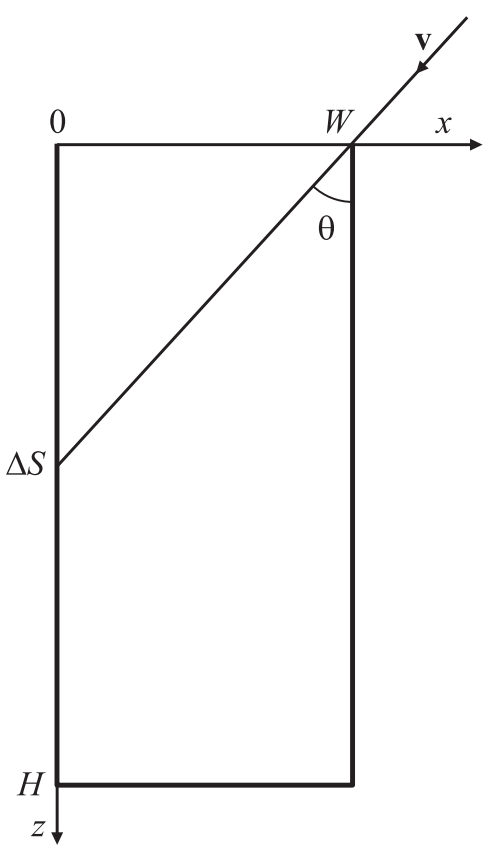

Pис. 1. Схема расчета плотности потока частиц из газовой фазы на боковую стенку тренча $\left(J_{e s}\right)$. 
полное число частиц $N$, получим

$$
\begin{aligned}
d \hat{N} & =v_{\perp} d t d S \frac{N}{V} \frac{d N_{v}}{N}=\left[\frac{N}{V}=n, \frac{d N_{v}}{N}=f(\mathbf{v} d \mathbf{v})\right] \\
& =v_{\perp} d S \operatorname{dtn} f(\mathbf{v}) d \mathbf{v} .
\end{aligned}
$$

Учитывая максвелловское распределение частиц по скоростям, получим плотность внешнего потока частиц на элемент поверхности боковой стенки. Согласно рис. 1, о площадку $\Delta S$ на боковой стенке тренча за время $\Delta t$ ударятся те частицы, направление скорости которых лежит в диапазоне углов $(0 ; \theta]\left(\operatorname{tg} \theta=\frac{W}{z}=\frac{v_{x}^{\max }}{v_{z}}\right)$. Плотность потока таких частиц определяется формулой

$$
\begin{aligned}
J_{e s} & =\frac{d N_{e}}{d S d t}=n_{g} \frac{m}{2 \pi k T_{g}} \int_{0}^{\infty} e^{-\frac{m v_{z}^{2}}{2 k T_{g}}} d v_{z} \int_{0}^{\frac{v_{z} W}{z}} v_{x} e^{-\frac{m v_{x}^{2}}{2 k T_{g}}} d v_{x} \\
& =\frac{n_{g}}{2 \pi} \int_{0}^{\infty}\left(1-e^{-\frac{m W^{2} v_{z}^{2}}{2 k z_{g}^{2}}}\right) e^{-\frac{m v_{z}^{2}}{2 k T_{g}}} d v_{z} \\
& =\frac{J_{f}}{2}\left(1-\left(1+\left(\frac{W}{z}\right)^{2}\right)^{-1 / 2}\right) \approx|W \ll z| \\
& \approx \frac{J_{f} W^{2}}{4 z^{2}}
\end{aligned}
$$

где $J_{f}=n_{g} \sqrt{\frac{m}{2 \pi k T_{g}}} \int_{0}^{\infty} v_{z} e^{-\frac{m v_{z}^{2}}{2 k T_{g}}} d v_{z}=n_{n} \sqrt{\frac{k T_{g}}{2 \pi m}}-$ плотность потока частиц на плоскую поверхность.

\section{2. Плотность потока частиц на дно тренча из газовой фазы $\left(J_{e b}\right)$}

Из рис. 2 видно, что о площадку $\Delta S$ на дне тренча за время $\Delta t$ ударятся те частицы, направление скорости которых лежит в диапазоне углов $\left[\theta_{1} ; \theta_{2}\right]\left(\operatorname{tg} \theta=\frac{x}{H}=\frac{v_{x}^{\max }}{v_{z}}\right)$. Выражение для плотности потока таких частиц имеет вид

$$
\begin{aligned}
J_{e b}= & n_{g} \frac{m}{2 \pi k T_{g}} \int_{0}^{\infty} v_{z} e^{-\frac{m v_{z}^{2}}{2 k T_{g}}} d v_{z} \int_{-\frac{v_{z} x}{H}}^{\frac{v_{z}(W-x)}{H}} e^{-\frac{m v_{x}^{2}}{2 k T_{g}}} d v_{x} \\
= & n_{g} \sqrt{\frac{m}{2 \pi k T_{g}}} \int_{0}^{\infty}\left(\operatorname{erf}\left(\frac{v_{z} x}{H} \sqrt{\frac{m}{2 k T_{g}}}\right)\right. \\
& \left.+\operatorname{erf}\left(\frac{v_{z}(W-x)}{H} \sqrt{\frac{m}{2 k T_{g}}}\right)\right) v_{z} e^{-\frac{m v_{z}^{2}}{2 k T_{g}}} d v_{z},
\end{aligned}
$$

где $\operatorname{erf}(z)-$ интеграл ошибок. Интегрируя по частям, получим окончательное выражение для плотности внеш-

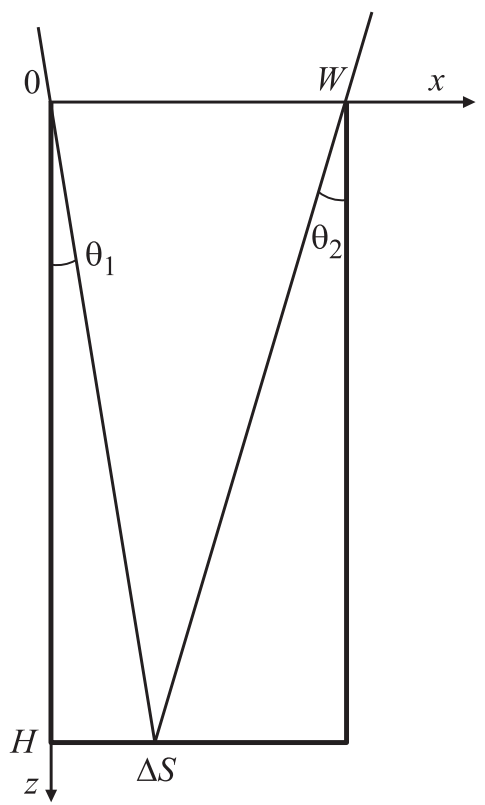

Рис. 2. Схема расчета плотности потока частиц из газовой фазы на дно тренча $\left(J_{e b}\right)$.

него потока частиц на дно тренча

$$
\begin{aligned}
J_{e b}= & \frac{J_{f}}{2}\left(\frac{x}{H}\left(1+\left(\frac{x}{H}\right)^{2}\right)^{-1 / 2}\right. \\
& \left.+\frac{W-x}{H}\left(1+\left(\frac{W-x}{H}\right)^{2}\right)^{-1 / 2}\right) \approx|W \ll z| \\
\approx & \frac{J_{f}}{2} \frac{W}{H}\left(1-\frac{W^{2}-3 x W+3 x^{2}}{2 H^{2}}\right) \approx \frac{J_{f}}{2 A},
\end{aligned}
$$

где $A=\frac{W}{H}-$ аспектное отношение.

Таким образом, видно, что при больших аспектных отношениях внешний поток на дно тренча значительно превышает поток на его боковую стенку вблизи дна. И, следовательно, дно станет являться источником отраженных частиц даже при коэффициенте прилипания $s=1$.

\section{3. Плотность потока частиц на стенки тренча за счет отражения от его дна $\left(J_{b s}\right)$}

Количество частиц, испускаемых площадкой $\Delta S$ в телесный угол $d \Omega$ под углом $\theta$ к нормали в единицу времени, определяется через поток частиц $\Phi$ выражением

$$
J=\frac{d \Phi}{d \Omega \Delta S_{\perp}}=\frac{d \Phi}{d \Omega \Delta S \cos \theta} \Rightarrow d \Phi=J d \Omega \Delta S \cos \theta .
$$

Для ламбертовского источника $J=$ const. Будем предполагать, что частицы при отражении от поверхности подчиняются закону Ламберта (закон косинуса).

Число молекул, ударившихся в единицу времени об элемент поверхности $d x d y$ на дне тренча, равно $J_{b}(x) \cdot d x d y$, где $J_{b}(x)$ - плотность потока частиц на 


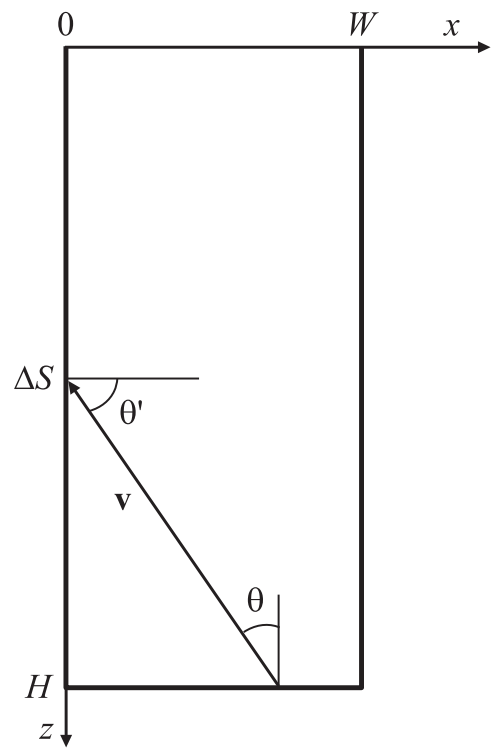

Pис. 3. Схема расчета плотности потока частиц на боковую стенку тренча за счет частиц, отраженных от его дна $\left(J_{b s}\right)$.

дно тренча (рис. 3). Из них внутри телесного угла $\Delta \Omega$ по направлению к площадке $\Delta S$, согласно закону косинуса, полетит $p(x, H) \cdot J_{b}(x) \cdot d x d y \frac{\Delta \Omega}{\pi} \cos \theta$ частиц.

Учитывая, что $\Delta \Omega=\frac{\Delta S \cos \theta^{\prime}}{(H-z)^{2}+x^{2}+y^{2}}$, после интегрирования по площади дна окончательно получим выражение для плотности потока частиц на площадку $\Delta S$ боковой стенки тренча за счет отраженных частиц от его дна

$$
\begin{aligned}
J_{b s}(z)= & \int_{0}^{W}(1-s(1-\Theta(x, H))) J_{b}(x) d x \\
& \times \int_{-\infty}^{\infty} d y \frac{\cos \theta^{\prime}}{(H-z)^{2}+x^{2}+y^{2}} \frac{1}{\pi} \cos \theta
\end{aligned}
$$

где $\cos \theta=\frac{H-z}{\sqrt{(H-z)^{2}+x^{2}+y^{2}}}, \cos \theta^{\prime}=\frac{x}{\sqrt{(H-z)^{2}+x^{2}+y^{2}}}$, а поток частиц на дно тренча $\left(J_{b}\right)$ определяется суммой внешнего потока $\left(J_{e b}\right)$ и потока, отраженного от боковых стенок $\left(J_{s b}\right)$ :

$$
\begin{aligned}
& J_{s b}(z)=\frac{1}{\pi}(H-z) \int_{0}^{W}(1-s(1-\Theta(x, H))) \cdot J_{b}(x) x d x \\
& \times \int_{-\infty}^{\infty} \frac{d y}{\left((H-z)^{2}+x^{2}+y^{2}\right)^{2}}=\frac{1}{2}(H-z) \\
& \times \int_{0}^{W}(1-s(1-\Theta(x, H))) \cdot J_{b}(x) \frac{x}{\left((H-z)^{2}+x^{2}\right)^{3 / 2}} d x .
\end{aligned}
$$

В результате получим выражение для потока $J_{b s}(z)$ :

$$
\begin{aligned}
J_{s b}(z) & =\frac{1}{2}(H-z) \int_{0}^{W}(1-s(1-\Theta(x, H))) \\
& \times\left(J_{e b}(x) \sqrt{\frac{T_{s}}{T_{g}}}+J_{s b}(x)\right) \frac{x}{\left((H-z)^{2}+x^{2}\right)^{3 / 2}} d x .
\end{aligned}
$$

Множитель $\sqrt{\frac{T_{s}}{T_{g}}}$ в скобках учитывает, что молекулы после удара о поверхность приобретают температуру подложки.

\section{4. Плотность потока частиц на дно тренча за счет отражения от его стенок $\left(J_{s b}\right)$}

Число молекул, ударившихся в единицу времени об элемент поверхности $d y d z$ боковой стенки тренча, равно $J_{s}(z) \cdot d y d z$, где $J_{s}(z)$ - плотность потока частиц на боковую стенку тренча (рис. 4). Из них внутри телесного угла $\Delta \Omega$ по направлению к площадке $\Delta S$, согласно закону косинуса, полетит $p(0, z) \cdot J_{s}(z) \cdot d y d x \frac{\Delta \Omega}{\pi} \cos \theta^{\prime}$ частиц.

Как и в предыдущем случае, элемент телесного угла определяется выражением $\Delta \Omega=\frac{\Delta S \cos \theta}{(H-z)^{2}+x^{2}+y^{2}}$. После интегрирования по $у$ и глубине тренча получим выражение для плотности потока частиц, отраженных от боковой

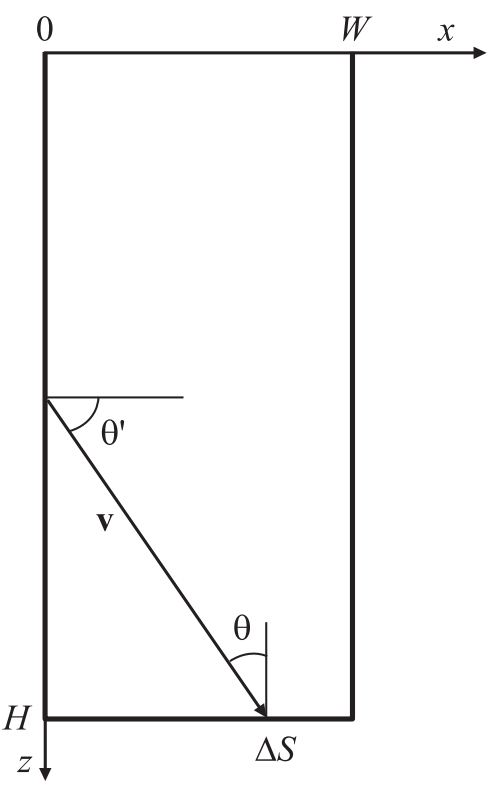

Рис. 4. Схема расчета плотности потока частиц на дно тренча за счет частиц, отраженных от его боковых стенок $\left(J_{s b}\right)$. 
стенки тренча с координатой $x=0$, на его дно

$$
\begin{gathered}
J_{s b}(x)=\frac{x}{\pi} \int_{0}^{H}(1-s(1-\Theta(0, z))) \cdot J_{s}(z)(H-z) d z \\
\quad \times \int_{-\infty}^{\infty} \frac{d y}{\left((H-z)^{2}+x^{2}+y^{2}\right)^{2}} \\
=\frac{x}{2} \int_{0}^{H}(1-s(1-\Theta(0, z))) J_{s}(z) \frac{(H-z)}{\left((H-z)^{2}+x^{2}\right)^{3 / 2}} d z,
\end{gathered}
$$

где поток частиц на стенку тренча $\left(J_{S}\right)$ определяется суммой внешнего потока $\left(J_{e s}\right)$, потока, отраженного от дна $\left(J_{b s}\right)$ и от противоположной стенки тренча $\left(J_{s s}\right)$. Отраженный поток на дно тренча от боковой стенки с координатой $x=W$ будет иметь тот же вид с заменой $x$ на $W-x$. В результате полный отраженный поток на дно тренча будет определяться суммой потоков от каждой из стенок

$$
\begin{aligned}
& J_{s b}(x)=\frac{x}{2} \int_{0}^{H}(1-s(1-\Theta(0, z))) \cdot J_{s}(0, z) \\
& \times \frac{(H-z)}{\left((H-z)^{2}+x^{2}\right)^{3 / 2}} d z+\frac{W-x}{2} \int_{0}^{H}(1-s(1-\Theta(W, z))) \\
& \times J_{s}(W, z) \frac{(H-z)}{\left((H-z)^{2}+(W-x)^{2}\right)^{3 / 2}} d z .
\end{aligned}
$$

Учитывая симметрию задачи, имеем $J_{s}(W, z)=J_{s}(0, z)$ и $\theta(W, z)=\theta(0, z)$, откуда получаем окончательное выражение для плотности потока частиц, отраженных от боковых стенок тренча на его дно:

$$
\begin{aligned}
& J_{s b}(x)=\frac{1}{2} \int_{0}^{H}(1-s(1-\Theta(0, z))) \\
& \quad \times\left(J_{e s}(z) \sqrt{\frac{T_{s}}{T_{g}}}+J_{b s}(z)+J_{s s}(z)\right) \\
& \quad \times\left[\frac{x}{\left((H-z)^{2}+x^{2}\right)^{3 / 2}}+\frac{W-x}{\left((H-z)^{2}+(W-x)^{2}\right)^{3 / 2}}\right] \\
& \quad \times(H-z) d z .
\end{aligned}
$$

\section{Плотность потока частиц на боковую стенку тренча за счет отражения от его противоположенной стенки $\left(J_{s s}\right)$}

Число молекул, ударившихся в единицу времени об элемент поверхности $d y d z_{1}$ боковой стенки, равно

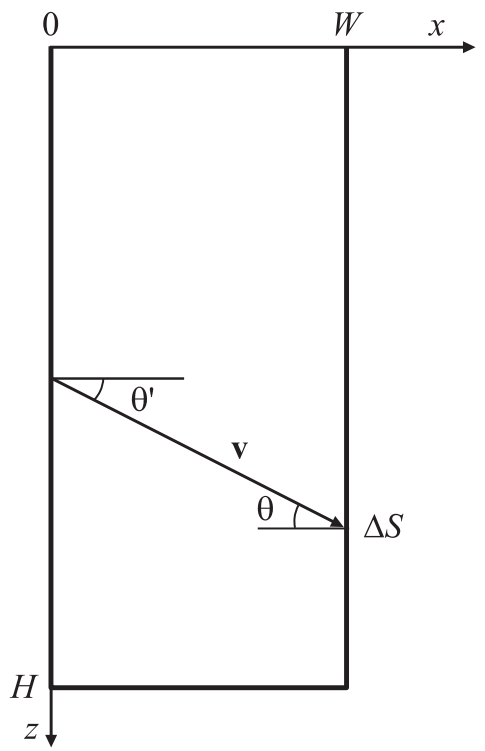

Рис. 5. Схема расчета плотности потока частиц на боковую стенку тренча за счет частиц, отраженных от его противоположенной стенки $\left(J_{s s}\right)$.

$J_{s}\left(z_{1}\right) \cdot d y d z_{1}$, где $J_{s}\left(z_{1}\right)$ - плотность потока частиц на боковую стенку тренча с координатой $z_{1}$ (рис. 5). Из них внутри телесного угла $\Delta \Omega$ по направлению к площадке $\Delta S$, находящейся на противоположенной стенке с координатой $z$, полетит $p\left(0, z_{1}\right) \cdot J_{s}\left(z_{1}\right) \cdot d y d z_{1} \frac{\Delta \Omega}{\pi} \cos \theta^{\prime}$ частиц.

Подставляя выражения для $\Delta \Omega=\frac{\Delta S \cos \theta}{\left(z-z_{1}\right)^{2}+W^{2}+y^{2}} \quad$ и $\cos \theta=\cos \theta^{\prime}=\frac{W}{\sqrt{\left(z-z_{1}\right)^{2}+W^{2}+y^{2}}}, \quad$ после интегрирования по $d z_{1}$ окончательно получим выражение для плотности потока частиц, отраженных от одной из боковых стенок и попавших на противоположную стенку на расстоянии $z$ от поверхности:

$$
\begin{aligned}
& J_{s s}(z)=\frac{W^{2}}{\pi} \int_{0}^{H}\left(1-s\left(1-\Theta\left(0, z_{1}\right)\right)\right) \cdot J_{s}\left(z_{1}\right) \cdot d z_{1} \\
& \times \int_{-\infty}^{+\infty} \frac{d y}{\left(\left(z-z_{1}\right)^{2}+W^{2}+y^{2}\right)^{2}}=\frac{W^{2}}{2} \int_{0}^{H}\left(1-s\left(1-\Theta\left(0, z_{1}\right)\right)\right) \\
& \times\left(J_{e s}\left(z_{1}\right) \sqrt{\frac{T_{s}}{T_{g}}}+J_{b s}\left(z_{1}\right)+J_{s s}\left(z_{1}\right)\right) \\
& \times \frac{d z_{1}}{\left(\left(z-z_{1}\right)^{2}+W^{2}\right)^{3 / 2}},
\end{aligned}
$$

где учтено, что поток частиц на стенку тренча $\left(J_{s}\right)$ определяется суммой внешнего потока $\left(J_{e s}\right)$, потока отраженного от дна $\left(J_{b s}\right)$ и от противоположенной стенки $\left(J_{S s}\right)$ тренча.

Таким образом, формула (6) с учетом полученных выражений для плотностей потоков частиц (9), (11), 
(15), (18), (19) представляет собой самосогласованное интегродифференциальное уравнение для определения степени заполнения поверхности тренча от времени подачи прекурсора.

\section{2. Оценка времени подачи прекурсора}

Получим приближенное выражение для времени, необходимого для однородного (в пределах 5\%) покрытия стенок тренча. Как показывают расчеты, при малых значениях коэффициента прилипания основной вклад в поток частиц на боковой стенке тренча вблизи его дна вносит поток, отраженный от противоположенной стенки. Подынтегральная функция в выражении для плотности потока отраженных от противоположенной стенки частиц $J_{s s}(z)$ обладает резким максимумом при $z_{1}=z$ и полушириной пика $\sim 2 W(19)$. Поэтому при больших $z$ можно приближенно записать

$$
\begin{aligned}
J_{s s}(z) & \approx(1-s(1-\Theta(0, z))) \\
& \times\left(J_{e s}(z) \sqrt{\frac{T_{s}}{T_{g}}}+J_{b s}(z)+J_{s s}(z)\right)\left(1-\frac{2 W^{2}}{z^{2}}\right),
\end{aligned}
$$

откуда получается приближенное выражение для отраженного от стенок тренча потока

$$
J_{s s}(z) \approx \frac{(1-s(1-\Theta(0, z)))\left(J_{e s}(z) \sqrt{\frac{T_{s}}{T_{g}}}+J_{b s}(z)\right)}{\frac{2 W^{2}}{z^{2}}+s(1-\Theta(0, z))} .
$$

Подставляя полученное выражение для плотности отраженного потока в кинетическое уравнение (6), получим приближенное уравнение для степени покрытия при $z^{\prime} \approx H$ :

$$
n_{0} \frac{d \Theta\left(0, z^{\prime}\right)}{d t} \approx \frac{\left(J_{e s}\left(z^{\prime}\right) \sqrt{\frac{T_{s}}{T_{g}}}+J_{b s}\left(z^{\prime}\right)\right)}{\frac{2}{A^{2}}+s\left(1-\Theta\left(0, z^{\prime}\right)\right)} s\left(1-\Theta\left(0, z^{\prime}\right)\right) .
$$

Для получения однородного покрытия (при учете самонасыщения реакций при ALD) необходимо, чтобы степень покрытия в любой точке поверхности тренча была не меньше, чем критическое значение $\Theta_{c}=0.95$. Выберем в качестве координаты $z^{\prime}$ точку, в которой поток имеет минимальное значение. Как показывают расчеты, данное значение соответствует величине $z^{\prime} \approx 0.8 H-0.95 H$ и зависит как от аспектного отношения, так и от коэффициента прилипания. Поскольку внешний поток ослабляется с глубиной по закону $\left(\frac{W}{z}\right)^{2}(9)$, то будем полагать, что

$$
J_{e s}\left(z^{\prime}\right) \sqrt{\frac{T_{s}}{T_{g}}}+J_{b s}\left(z^{\prime}\right) \sim J_{f}\left(\frac{W}{z^{\prime}}\right)^{2} \sim \frac{J_{f}}{A^{2}} .
$$

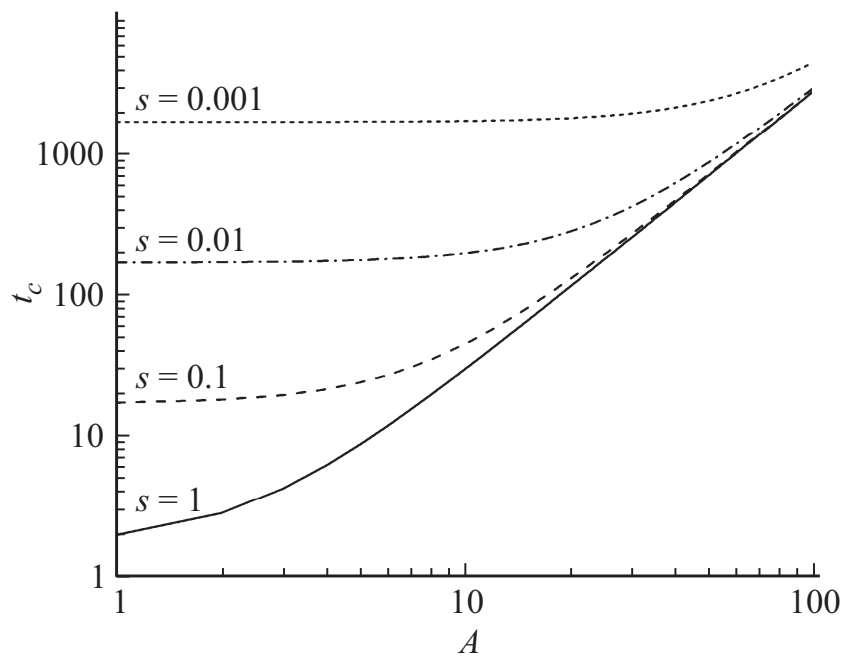

Рис. 6. Аналитическая зависимость времени, необходимого для однородного покрытия стенок тренча, от аспектного отношения при различных значениях коэффициента прилипания, рассчитанная по формуле (24).

С учетом (23) после интегрирования получим выражение для времени, необходимого для заполнения поверхности тренча до значения $\Theta_{c}$ (критическое время):

$$
t_{c}(A, s) \approx \alpha \frac{n_{0} A^{2}}{s J_{f}}\left(s+\frac{2}{A^{2}} \ln \left(\frac{1}{\left(1-\Theta_{c}\right)}\right)\right),
$$

где $\alpha-$ нормировочный множитель.

Согласно полученной формуле, критическое время имеет две асимптотики в зависимости от преобладания одного из слагаемых в скобках. При малых значениях коэффициента прилипания критическое время перестает зависеть от аспектного отношения и определяется коэффициентом прилипания $t_{c} \propto s$. В обратном случае критическое время слабо зависит от коэффициента прилипания и определяется аспектным отношением $t_{c} \propto A^{-2}$. Этот случай согласуется с результатами работы [4], где критическое время было получено для коэффициента прилипания $s=1$.

Рассчитанная по формуле (24) зависимость критического времени от аспектного отношения при различных значениях коэффициента прилипания представлена на рис. 6. Время нормировалось на время, необходимое для покрытия плоской поверхности при значении коэффициента прилипания $s=1$. Эти кривые согласуются с результатами работы [3], полученными методом МонтеКарло.

\section{3. Определение профиля покрытия стенок}

Во втором разделе были получены выражения для плотностей потоков на элемент поверхности тренча как 
частиц, попадающих из газовой фазы, так и частиц, отраженных боковыми стенками и дном тренча (15), (18), (19). Используя указанные выражения, можно записать систему самосогласованных интегродифференциальных уравнений:

$\left\{\begin{array}{l}n_{0} \frac{d \Theta(0, z)}{d t}=\left(J_{e s}(z)+J_{s s}(z)+J_{b s}(z)\right) s(1-\Theta(0, z)), \\ n_{0} \frac{d \Theta(x, H)}{d t}=\left(J_{e b}(x)+J_{s b}(x)\right) s(1-\Theta(0, z)) .\end{array}\right.$

Будем решать систему (25) численно. Для этого временной интервал разобьем на отрезки $\Delta t$, определяемые как время, необходимое для заполнения плоской поверхности на $\eta=1 \%$,

$$
n_{0} \frac{d \Theta_{f}}{d t}=J_{f} s\left(1-\theta_{f}\right) \Rightarrow \Delta t=-\frac{n_{0}}{s J_{f}} \ln (1-\eta) .
$$

На первом временном интервале $[0 ; \Delta t]$ будем пренебрегать отраженными потоками в (25), т.е. система уравнений примет вид

$$
\left\{\begin{array}{l}
n_{0} \frac{d \Theta(0, z)}{d t}=s(1-\Theta(0, z)) J_{e s}(z) \\
n_{0} \frac{d \Theta(x, H)}{d t}=s(1-\Theta(x, H)) J_{e b}(x) .
\end{array}\right.
$$

Полученные решения для степени заполнения поверхности подставляются в выражения для плотностей отраженных потоков

$$
\begin{aligned}
& J_{b s}(z)=\frac{1}{2}(H-z) \sqrt{\frac{T_{s}}{T_{g}}} \int_{0}^{W}(1-s(1-\Theta(x, H))) \\
& \times J_{e b}(x) \frac{x}{\left((H-z)^{2}+x^{2}\right)^{3 / 2}} d x \\
& J_{s b}(x)=\frac{1}{2} \sqrt{\frac{T_{s}}{T_{g}}} \int_{0}^{H}(1-s(1-\Theta(0, z))) J_{e s}(z) \\
& \times\left[\frac{x}{\left((H-z)^{2}+x^{2}\right)^{3 / 2}}+\frac{\left((H-z)^{2}+(W-x)^{2}\right)^{3 / 2}}{(H-x) d z,}\right. \\
& \times(H) \\
& J_{s s}(z)=\frac{W^{2}}{2} \sqrt{\frac{T_{s}}{T_{g}}} \int_{0}^{H}(1-s(1-\Theta(0, z))) J_{e s}\left(z_{1}\right) \\
& \times \frac{d z_{1}}{\left(\left(z-z_{1}\right)^{2}+W^{2}\right)^{3 / 2}} .
\end{aligned}
$$

На следующем временном интервале $[\Delta t ; 2 \Delta t]$ определяются степень заполнения поверхности тренча (25), а также плотности отраженных потоков (15), (18), (19). При этом в правую часть выражений подставляются степень заполнения и значения отраженных потоков,

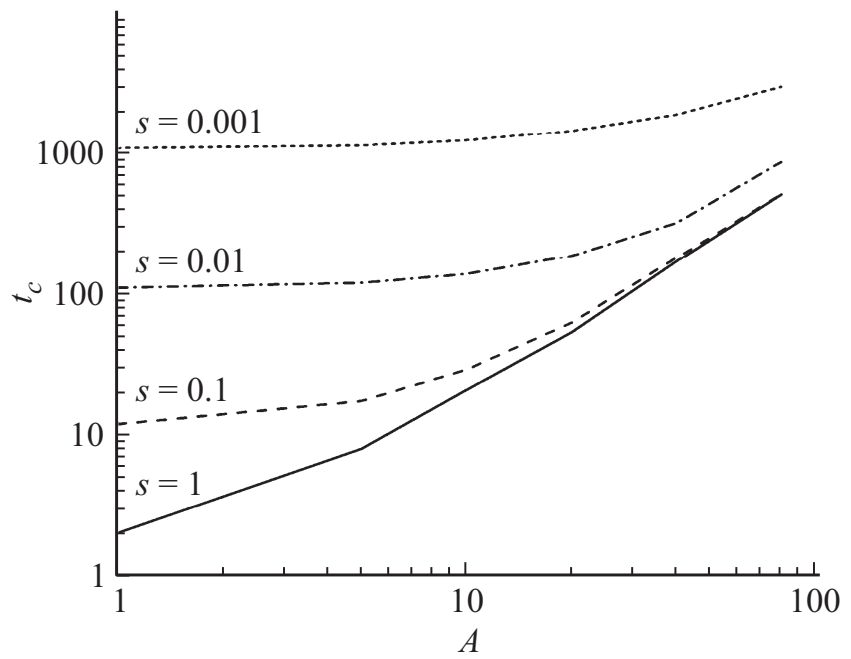

Рис. 7. Расчетная зависимость времени, необходимого для однородного покрытия стенок тренча, от аспектного отношения при различных значениях коэффициента прилипания.

полученные на предыдущем шаге. Далее процесс повторяется до полного заполнения поверхности, или исчерпания заданного времени подачи прекурсора.

С использованием описанного алгоритма были рассчитаны времена, необходимые для однородного покрытия стенок тренча на 95\%. Результаты вычислений приведены на рис. 7. Время нормировалось на время, необходимое для покрытия плоской поверхности при значении коэффициента прилипания $s=1$. Как можно видеть, характер зависимости критического времени покрытия стенок тренча от аспектного отношения соответствует результатам, полученным по приближенной формуле (рис. 6). Однако по результатам численного расчета получается более плавный рост кривых и выход на асимптотику при больших значениях аспектного отношения.

\section{Заключение}

В работе развит последовательный подход, основанный на применении кинетических уравнений, описывающих степень заполнения элемента поверхности тренча в зависимости от времени подачи прекурсора. Потоки частиц на стенки тренча в рассматриваемом подходе определяются согласно формулам молекулярнокинетической теории.

Получена аналитическая формула, позволяющая оценить критическое время, необходимое для однородного покрытия стенок тренча в зависимости от аспектного отношения тренча и коэффициента прилипания молекул прекурсора. Предложен алгоритм численного расчета, позволяющий предсказать пространственную форму профиля нанесенной пленки при заданном времени подачи прекурсора, а также рассчитать время, необходимое для однородного покрытия стенок тренча. Согласно 
полученным результатам, в кривых, описывающих зависимость критического времени покрытия тренча от аспектного отношения при различных значениях коэффициента прилипания, наблюдаются два асимптотических режима, отвечающих различным предельным случаям. При малом значении коэффициента прилипания $\left(s \ll A^{-2}\right)$ критическое время подачи прекурсора для однородного покрытия поверхности тренча примерно равно времени заполнения плоской поверхности и не зависит от аспектного отношения тренча. При обратном соотношении критическое время пропорционально квадрату аспектного отношения и фактически не зависит от коэффициента прилипания.

Результаты численного расчета и аналитическая зависимость качественно согласуются как между собой, так и с результатами других авторов [3], полученными методом Монте-Карло.

Полученный результат позволяет оптимизировать процессы атомно-слоевого осаждения в выскоаспектные структуры не только по времени экспозиции прекурсора, но и по коэффициенту прилипания за счет выбора температуры подложки. Снижение коэффициента прилипания позволяет получить более однородное покрытие стенок глубоких тренчей при равных длительностях процесса. Для оценки времени экспозиции может быть использована полученная впервые аналитическая формула (24).

\section{Список литературы}

[1] Atomic Layer Deposition of Nanostructured Materials / Ed. by N. Pinna, M. Knez. Wiley-VCH Verlag \& Co. KGaA, 2012. $435 \mathrm{p}$.

[2] Rose M., Bartha J.W. // Appl. Surface Sci. 2009. Vol. 255. P. 6620-6623.

[3] Knoops H.C.M., Langereis E,. vandeSanden M.C.M, Kessels M.M. // J. Electrochem. Soc. 2010. Vol. 157. N 12. P. G241-G249.

[4] Gordon R.G., Hausmann D., Kim E., Shepard J. // Chem. Jap. Deposition. 2003. Vol. 9. N 2. P. 73-78.

[5] Yanguas-Gil A., Elam J.W. // ECS Transactions. 2011. Vol. 41. N 2. P. $169-174$.

[6] Yanguas-Gil A., Elam J.W. // Chem. Vap. Deposition. 2012. Vol. 18. P. 46-52.

[7] Wang W.B., Abelson J.R. // J. Appl. Phys. 2014. Vol. 116. P. $116-124$. 